\title{
Gastroblastoma harbors a recurrent somatic MALAT1-GLI1 fusion gene
}

Rondell P Graham ${ }^{1,2}$, Asha A Nair ${ }^{3}$, Jaime I Davila ${ }^{3}$, Long Jin ${ }^{2}$, Jin Jen ${ }^{4}$, William R Sukov ${ }^{2}$, Tsung-Teh $\mathrm{Wu}^{1}$, Henry D Appelman ${ }^{5}$, Jorge Torres-Mora ${ }^{1}$, Kyle D Perry ${ }^{1}$, Lizhi Zhang ${ }^{1}$, Sara M Kloft-Nelson ${ }^{4}$, Ryan A Knudson ${ }^{4}$, Patricia T Greipp ${ }^{2}$ and Andrew L Folpe ${ }^{1}$

${ }^{1}$ Division of Anatomic Pathology, Department of Laboratory Medicine and Pathology, Mayo Clinic, Rochester, MN, USA; ${ }^{2}$ Division of Laboratory Genetics/Genomics, Department of Laboratory Medicine and Pathology, Mayo Clinic, Rochester, MN, USA; ${ }^{3}$ Department of Health Sciences Research, Mayo Clinic, Rochester, MN, USA; ${ }^{4}$ Medical Genome Facility, Mayo Clinic, Rochester, MN, USA and ${ }^{5}$ Department of Pathology, University of Michigan, Ann Arbor, MI, USA

\begin{abstract}
Gastroblastoma is a rare distinctive biphasic tumor of the stomach. The molecular biology of gastroblastoma has not been studied, and no affirmative diagnostic markers have been developed. We retrieved two gastroblastomas from the consultation practices of the authors and performed transcriptome sequencing on formalin-fixed paraffin-embedded tissue. Recurrent predicted fusion genes were validated at genomic and RNA levels. The presence of the fusion gene was confirmed on two additional paraffin-embedded cases of gastroblastoma. Control cases of histologic mimics (biphasic synovial sarcoma, leiomyoma, leiomyosarcoma, desmoid-type fibromatosis, EWSR1-FLI1-positive Ewing sarcoma, Wilms' tumor, gastrointestinal stromal tumor, plexiform fibromyxoma, Sonic hedgehog-type medulloblastomas, and normal gastric mucosa and muscularis propria were also analyzed. The gastroblastomas affected two males and two females aged 9-56 years. Transcriptome sequencing identified recurrent somatic MALAT1-GL/1 fusion genes, which were predicted to retain the key domains of GLI1. The MALAT1-GLI1 fusion gene was validated by break-apart and dual-fusion FISH and RT-PCR. The additional two gastroblastomas were also positive for the MALAT1-GLI1 fusion gene. None of the other control cases harbored MALAT1-GLI1. Overexpression of GLI1 in the cases of gastroblastomas was confirmed at RNA and protein levels. Pathway analysis revealed activation of the Sonic hedgehog pathway in gastroblastoma and gene expression profiling showed that gastroblastomas grouped together and were most similar to Sonic hedgehog-type medulloblastomas. In summary, we have identified an oncogenic MALAT1-GLI1 fusion gene in all cases of gastroblastoma that may serve as a diagnostic biomarker. The fusion gene is predicted to encode a protein that includes the zinc finger domains of GLI1 and results in overexpression of GLI1 protein and activation of the Sonic hedgehog pathway.
\end{abstract}

Modern Pathology (2017) 30, 1443-1452; doi:10.1038/modpathol.2017.68; published online 21 July 2017

Gastroblastoma is an extremely rare, distinctive, biphasic tumor of the stomach. To date, only eight cases have been reported, all in patients under 30 years of age. ${ }^{1-6}$ Owing to its biphasic morphology, gastroblastoma may mimic other biphasic tumors of the stomach (eg, synovial sarcoma and sarcomatoid carcinoma). In smaller biopsies that do not demonstrate biphasic morphology, the differential diagnosis of gastroblastoma is broad, and includes a

Correspondence: Dr AL Folpe, MD, Division of Anatomic Pathology, Department of Laboratory Medicine and Pathology, Mayo Clinic, 200 First Street SW, Rochester, MN 55905, USA.

E-mail: Folpe.Andrew@Mayo.edu

Received 1 April 2017; revised 1 May 2017; accepted 2 May 2017; published online 21 July 2017 wide variety of other gastric tumors characterized by relatively bland epithelioid or spindled cells, including the recently described plexiform fibromyxoma, a rare gastric spindle cell neoplasm recently shown to harbor a MALAT1-GLI1 fusion gene in $\sim 15-20 \%$ of cases. $^{7}$

The molecular pathogenesis of gastroblastoma is unknown, and there are currently no ancillary tests that are known to be useful in its differential diagnosis with other gastric neoplasms.

We hypothesized that the distinctive clinical and morphological features of gastroblastoma suggested a translocation-associated neoplasm, and for this reason we studied the morphological, immunohistochemical, and molecular genetic features of a series of these rare tumors. 


\section{Materials and methods}

\section{Cases}

All available routinely stained slides, immunohistochemical studies, and formalin-fixed paraffinembedded tissue blocks from four cases of gastroblastoma were retrieved from our collective consultation files and re-reviewed by two of the authors (RPG and ALF). The clinicopathological features of two of these cases have been previously published (case nos. 1 and 3)..$^{2,3}$ Clinical information including patient age, sex, tumor size, metastases, and clinical follow-up was obtained whenever possible.

Formalin-fixed paraffin-embedded tissue blocks from cases of potential histologic mimics (controls) were also selected. The controls included the following: biphasic synovial sarcoma $(n=7)$; leiomyoma $(n=5)$; leiomyosarcoma $(n=5)$; desmoid-type fibromatosis $(n=5)$; EWSR1-FLI1-positive Ewing sarcoma $(n=5)$; Wilms' tumor $(n=2)$; plexiform fibromyxoma $(n=2)$; gastrointestinal stromal tumor $(n=2)$; and normal gastric muscularis propria $(n=1)$.

\section{RNA Sequencing}

Global transcriptome sequencing was performed on formalin-fixed paraffin-embedded tissue from two gastroblastomas (case nos. 1 and 2) and one sample of randomly selected normal gastric muscularis propria. Total RNA libraries were prepared from each case (Illumina TruSeq RNA Access) and run on the Illumina High Seq-2000 for $101 \mathrm{bp}$ paired-end reads. Gene expression counts were obtained using the MAP-RSeq v.2.0.0 workflow. MAP-RSeq consists of alignment with TopHat 2.0.12 against the human hg19 genome build and gene counts with the Subread package 1.4.4. Gene annotation files were obtained from Ensemble version 75. Gene counts were normalized using the reads per kilobase per million mapped reads approach. Differential expression analysis was performed using edgeR 2.6.2. Gene fusions were identified using TopHat-Fusion ${ }^{8}$ and RNA sequencing (RNASeq) quality was assessed with Fusion Sense. ${ }^{9}$

\section{Pathway Analysis}

Enriched canonical pathways for differentially expressed genes obtained from the RNASeq data were investigated using the Ingenuity pathway analysis software IPA (Ingenuity Systems, www. ingenuity.com). Canonical pathways, biological functions, and networks were used for critical investigation of cancer-related pathways.

\section{Reverse Transcriptase-PCR and Fluorescence In Situ Hybridization}

Reverse transcriptase-PCR (RT-PCR) and fluorescence in situ hybridization (FISH) were used to validate recurrent predicted fusion genes from RNASeq analysis at transcriptional and genomic levels, respectively, in all four cases. RT-PCR was performed on case nos. 1 and 2 as follows: $500 \mathrm{ng}$ of RNA from formalin-fixed paraffin-embedded tissue was converted into cDNA in a $20 \mu \mathrm{l}$ of reaction volume using the iScript Select cDNA Synthesis kit (Bio-Rad Laboratories, Hercules, CA, USA). The MALAT1-GLI1 fusion genes (Genbank accession no.: MALAT1: NR_002819.3; GLI1: NM_005269.2) were validated using multispecific PCR primers. For case no. 1, the following primer sequences were utilized: 5'-AGGTTACTAAGATATTGCTTAGCGT-3' (forward) and 5'-CAGACTTCAGCTTCCCCAGG-3' (reverse). For case no. 2, primer sequences 5'-GGTT ACTAAGATATTGCTTAGCGTT-3' (forward) and 5'-CCTGAGCTGAGGGAAATCAGG-3' (reverse) were utilized. The housekeeping gene phosphoglycerokinase (PGK1; NM_000291.3) was amplified along with MALAT1-GLI1 fusion transcripts to check for the RNA quality, using a primer set 5'-CAGTTTGGAGCT CCTGGAAG-3' (forward) and 5'-CTGGCACTGCATCT CTTGGCC-3' (reverse). PCR was carried out in a $25 \mu \mathrm{l}$ final reaction volume containing $2 \mu \mathrm{l}$ of cDNA template using the conditions previously reported by our group. ${ }^{10}$ After amplification, the PCR products were visualized by $3 \%$ agarose gel electrophoresis. No reverse transcriptase in $\mathrm{RT}$ reaction was used as negative control. All positive RT-PCR products were confirmed by Sanger sequencing.

FISH was done for the rearrangement of the MALAT1 locus, the GLI1 locus, and the fusion of MALAT1-GLI1 using lab-developed protocols at the Mayo Clinic. MALAT1 break-apart (BAP) and MALAT1-GLI1 dual-fusion FISH (D-FISH) were done on all four gastroblastomas and both plexiform fibromyxomas. GLI1 BAP FISH was done on all four gastroblastomas and all controls.

For MALAT1 BAP FISH, human bacterial artificial chromosomes (BACs) flanking the MALAT1 locus were identified using the University of California Santa Cruz February 2009 Assembly hg19. The 5'-MALAT1 clones (RP11-399J13, RP11-251P4, and RP11-436C17) were labeled by nick translation with Spectrum Green dUTP (Abbott Molecular/Vysis Products, IL) and the 3'-MALAT1 clones (RP11-472D14 and RP11-263H6) were labeled with Spectrum Orange dUTP (Abbott Molecular/Vysis Products). Labeled clones were combined to create a dual-color BAP probe set.

For GLI1 BAP FISH, human BACs flanking the GLI1 locus were identified in a similar manner to the above. The 5'-GLI1 clones (RP11-57K19, RP11756H6, and WI2-684I4) were labeled as above with Spectrum Orange dUTP (Abbott Molecular/Vysis Products) and the $3^{\prime}-$-GLI1 clones (WI2-2018I10, WI2-1865J18, and RP11-571M6) were labeled with Spectrum Green dUTP (Abbott Molecular/Vysis Products). Labeled clones were combined to create a dual-color BAP probe set.

For MALAT1-GLI1 D-FISH, human BACs spanning both the MALAT1 and GLI1 loci were identified 
similar to the above. The MALAT1 clones (RP111104L6 and RP11-472D15) were labeled by with Spectrum Orange dUTP (Abbott Molecular/ Vysis Products) and the GLI1 clones (RP11-57K19, RP11-258J5, and RP11-181L23) were labeled with Spectrum Green dUTP (Abbott Molecular/Vysis Products). Labeled clones were combined to create a D-FISH probe set.

Each probe set (MALAT1 BAP, GLI1 BAP, and MALAT1-GLI1 D-FISH) was applied to individual $5 \mu \mathrm{m}$ formalin-fixed paraffin-embedded unstained slides, hybridized, and washed according to a previously published FISH protocol. ${ }^{11}$

\section{Gene Expression Profiling}

Gene expression profiling was performed on RNA derived from formalin-fixed paraffin-embedded tissue of case nos. 1 and 2 of gastroblastoma along with six leiomyomata, three desmoid-type fibromatosis, three Sonic hedgehog-type medulloblastomas, two biphasic synovial sarcomas, two Wilms' tumors, and two plexiform fibromyxomas as controls. Transcriptome profiles were measured using Affymetrix Human Transcriptome Array 2.0 (http://www.affy metrix.com). Gene-level normalization and signal summarization were done using the Affymetrix Expression Console (Affymetrix). To study the proximity of tumors on the basis of their expression profiles, probes with the highest variance across all tumors were first selected. Next, unsupervised hierarchical clustering was performed on the normalized data using heatmap. 2 using euclidean distance and ward.D2 linkage metrics.

\section{Immunohistochemistry}

GLI1 immunohistochemistry was performed on $5 \mu \mathrm{m}$ unstained sections of four cases of gastroblastoma, two examples of Sonic Hedgehog-type medulloblastoma, and the following controls: seven biphasic synovial sarcomas; five desmoid-type fibromatosis; five leiomyomas; five leiomyosarcomas; five Ewing sarcomas, two Wilms' tumors, and two plexiform fibromyxomas using clone C-1 (Santa Cruz Biotechnology, Dallas, TX, USA), dilution 1:50 and EDTA antigen retrieval for 20 min. Antibody incubation and detection for GLI1 were done on the Leica BOND RX staining platform (pre-treatment BOND ER 2) with detection using a Leica BOND Refine Polymer Detection System, DS9800 (Leica Biosystems). Normal cerebellum and normal liver were used as positive and negative controls, respectively.

\section{Results}

\section{Clinical Features}

Table 1 summarizes the clinicopathological features of the cases in this study. The gastroblastomas arose in two males and two females aged 9-56 years (mean $30 \pm 19$ years). The tumors were centered in the stomach with three cases involving the antrum. In two patients, metastases were present at the time of consultation. The metastases involved a perigastric lymph node, peritoneum, liver, and urinary bladder in one case (case no. 1) and the liver in the other case (case no. 4). Three cases were resection specimens and the fourth (case no. 4) was a needle biopsy from the stomach.

\section{Histologic and Immunohistochemical Features}

Histologically, all tumors displayed biphasic morphology. Two cases showed relatively even proportions of epithelial and spindled elements, whereas the other two cases were composed largely of epithelial cells. The epithelial elements of all tumors were composed of nests of uniform epithelioid cells with scant amounts of pale eosinophilic cytoplasm and round to oval, normochromic nuclei. Within these tumor nests, rosette-like structures with central eosinophilic material were frequently present. The spindle cell component was characterized by bland, elongated, slender cells separated by a loose myxoid matrix, creating a reticular appearance. Mitotic figures were occasionally seen and were normal in appearance (Figure 1a-f). Histologic sections from a lymph node metastasis were available for case no. 1, and showed features similar to the primary tumor (Figure 1b). The metastatic lesions for case no. 4 were radiographically noted, but not biopsied.

Table 2 summarizes the immunohistochemical results. The epithelial component was positive for pan-keratins AE1/AE3 or OSCAR in all cases and was consistently negative for chromogranin and synaptophysin. The spindle component was positive

Table 1 Summary of the clinical characteristics of the cases of gastroblastoma

\begin{tabular}{lcclclc}
\hline Case & Age & Sex & Specimen type & Tumor size $(\mathrm{cm})$ & Metastases & Follow-up (mos) \\
\hline 1 & 28 & M & Resection & 3.8 & Lymph node, liver, peritoneum & N/A \\
2 & 27 & M & Resection & & No & N/A \\
3 & 9 & M & Resection & 9.0 & No & ANED \\
4 & 56 & F & Needle Bx & 4.0 & Liver & ANED \\
& & & & & N/A \\
\hline
\end{tabular}

Abbreviations: ANED: alive no evidence of disease; N/A: not available. 

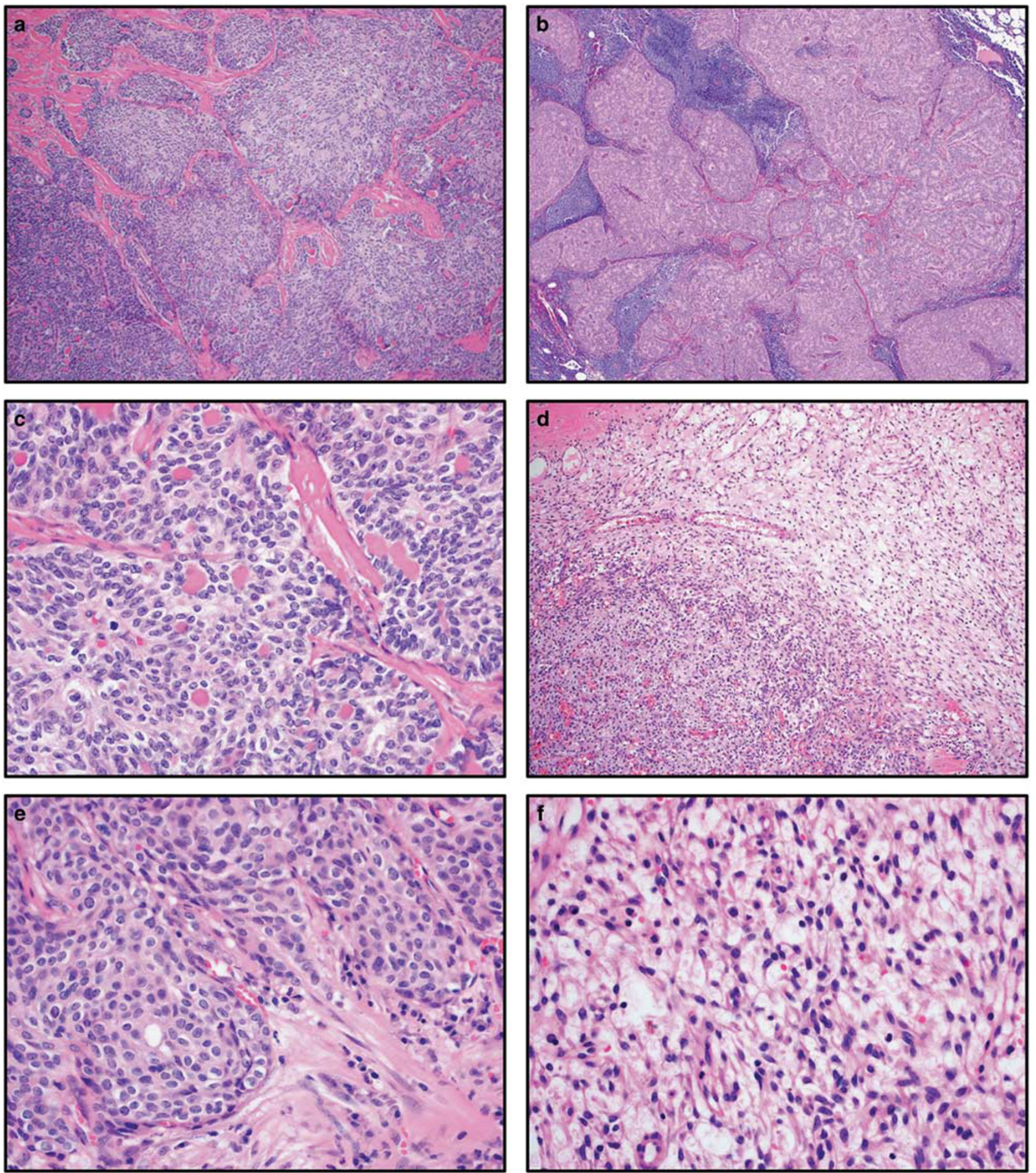

Figure 1 (a) Gastroblastoma showing a nested multinodular growth pattern. (b) Lymph node metastasis from a gastroblastoma (case 1). (c) Areas resembling rosette-like structures may be seen in gastroblastoma as shown in this figure. (d) A biphasic growth pattern as shown in this panel is the most characteristic pattern of gastroblastoma (case 2). (e) The epithelial component is composed of nests of small blandappearing cells. (f) The spindle component of gastroblastoma often shows a reticular pattern and similar to the epithelial component is bland appearing.

for vimentin in both cases where this marker was used. One case showed patchy smooth muscle actin expression but smooth muscle actin was negative in two other cases.

\section{RNA Sequencing}

RNA sequencing (RNASeq) followed by fusion gene analysis identified a recurrent, fusion of MALAT1 
Table 2 Summary of the immunohistochemical results of the cases of gastroblastoma

\begin{tabular}{|c|c|c|}
\hline Case & Positive immunohistochemistry results & Negative immunohistochemistry results \\
\hline 1 & $\mathrm{AE} 1 / \mathrm{AE}^{\mathrm{e}}, \mathrm{CD}^{\mathrm{e}}{ }^{\mathrm{e}}, \mathrm{NSE}^{\mathrm{e}}$, low Ki-67 ( 10\%) & Chromogranin, synaptophysin, CEA, TTF-1,PLAP, CD30, AFP, HCG \\
\hline 2 & $\mathrm{AE} 1 / \mathrm{AE}^{\mathrm{e}}$, patchy $\mathrm{SMA}^{\mathrm{s}}$ & $\begin{array}{l}\text { Chromogranin, synpatophysin, KIT, DOG1, desmin, S100, melan-A, SOX10, } \\
\text { TLE-1, CD99, keratin 5/6 }\end{array}$ \\
\hline 3 & $\begin{array}{l}\mathrm{AE} 1 / \mathrm{AE} 3^{\mathrm{e}} \text {, focal CD10 } \\
\text { vimentin }^{\mathrm{s}} \text {, KIT, } \mathrm{CD} 56^{\mathrm{e}} \text {, }\end{array}$ & $\begin{array}{l}\text { Chromogranin, synaptophysin, NSE, desmin, SMA, calretinin, inhibin, KRT } \\
\text { 34ßE12, CD34 }\end{array}$ \\
\hline 4 & $\begin{array}{l}\text { Patchy OSCAR } \text {, low Ki-67 ( 10\%), } \\
\text { vimentins }^{\text {s }}\end{array}$ & $\begin{array}{l}\text { KRT 34 } \beta \text { E12, KRT7, KRT 20, CDX2, chromogranin, synaptophysin, CD34, CD99, } \\
\text { KIT, DOG1, calretinin, WT1, SMA, desmin, EMA, MOC31, melan-A, HMB-45, } \\
\text { pCEA }\end{array}$ \\
\hline
\end{tabular}

Immunohistochemical markers apply to both epithelial and spindle cell components unless denoted as follows:

${ }^{\text {e}}$ refers to positive expression in the epithelial component only.

${ }^{s}$ refers to positive expression in the spindle cell component only.

and GLI1 in both cases (case nos. 1 and 2). No other fusion genes were identified. In case no. 1, MALAT1 exon 1 was fused to GLI1 intron 5 to exon 12 , and in case no. 2, MALAT1 exon 1 was fused to GLI1 exons 5-12 (Supplementary Figure 1). In both cases, a novel transcription start site is created at GLI1 exon 6. A reciprocal GLI1-MALAT1 fusion gene was identified in both analyzed cases but both reciprocal GLI1-MALAT1 transcripts included premature stop codons. No fusion genes were identified in the normal gastric wall control evaluated by RNASeq. A table showing the quality metrics of RNASeq is included in the Supplementary Data (Supplementary Table 1).

\section{Reverse Transcriptase-PCR}

RT-PCR confirmed the presence of the MALAT1GLI1 fusion transcript in both case nos. 1 and 2 of gastroblastoma (Figure 2a). The lymph node metastasis from case no. 1 was also positive for the MALAT1-GLI fusion transcript. Sanger sequencing of the cDNA from case nos. 1 and 2 confirmed fusion of MALAT1 to GLI1 (Figure 2b). RT-PCR failed for cases 3 and 4, owing to nucleic acid degradation as evidenced by lack of amplification of the internal control PGK.

\section{Fluorescence In Situ Hybridization}

MALAT1 BAP, GLI1 BAP, and MALAT1-GLI1 D-FISH were positive for rearrangement of the MALAT1 and GLI1 loci and fusion of the MALAT1 and GLI1 loci, respectively, in all four studied cases (Figure 3a and b) in both the epithelial and spindle cell components.

\section{RT-PCR and FISH Results in Morphological Mimics}

Potential histologic mimics, including seven biphasic synovial sarcomas, two gastrointestinal stromal tumors, five leiomyomas, five leiomyosarcomas, five desmoid-type fibromatoses, five Ewing sarcomas, two Wilms' tumors, and two plexiform fibromyxomas were also studied by RT-PCR and FISH. One plexiform fibromyxoma was positive for MALAT1 rearrangement but negative for GLI1 rearrangement and MALAT1-GLI1 D-FISH (data not shown). The other plexiform fibromyxoma was negative for each of the FISH strategies. All of the other controls were negative for MALAT1-GLI1 by RT-PCR and were negative for GLI1 BAP FISH.

\section{RNASeq Analysis}

By RNASeq analysis of case nos. 1 and 2, GLI1 and its known target genes PTCH1, SOX2, VEGFA, and CCND1 were differentially overexpressed in gastroblastoma with a greater than eightfold change $(P<0.004$ for each) compared to normal gastric muscularis propria (Supplementary Table 2). This indicates that the MALAT1-GLI1 fusion transcript results in the expression of GLI1. Pathway analysis on these cases looking at differentially expressed genes showing a greater than threefold change and a false discovery rate $<0.05$ from this data set identified upregulation of the Sonic hedgehog pathway (including GLI1, HHIP, PTCH1, and PTCH2) in the tumor cells of gastroblastoma $(P=0.013)$ and downregulation of the protein kinase A pathway, an antagonist of Sonic hedgehog pathway signaling $\left(P=4.57 \times 10^{-8}\right)$.

\section{GLI1 Immunohistochemistry}

We confirmed GLI1 overexpression by immunohistochemistry, showing that GLI1 was strongly and diffusely positive in a nuclear and cytoplasmic pattern in three of three cases of gastroblastoma (Figure 4). Because of our finding of upregulation of the Sonic Hedgehog pathway in gastroblastoma (above), we also studied two cases of Sonic Hedgehog-type medulloblastomas, both of which were also diffusely positive. The remaining controls (seven synovial sarcomas, five desmoid-type fibromatoses, five leiomyomas, five leiomyosarcomas, five Ewing sarcomas, two Wilms' tumors, and two plexiform fibromyxomas) were negative. Review 

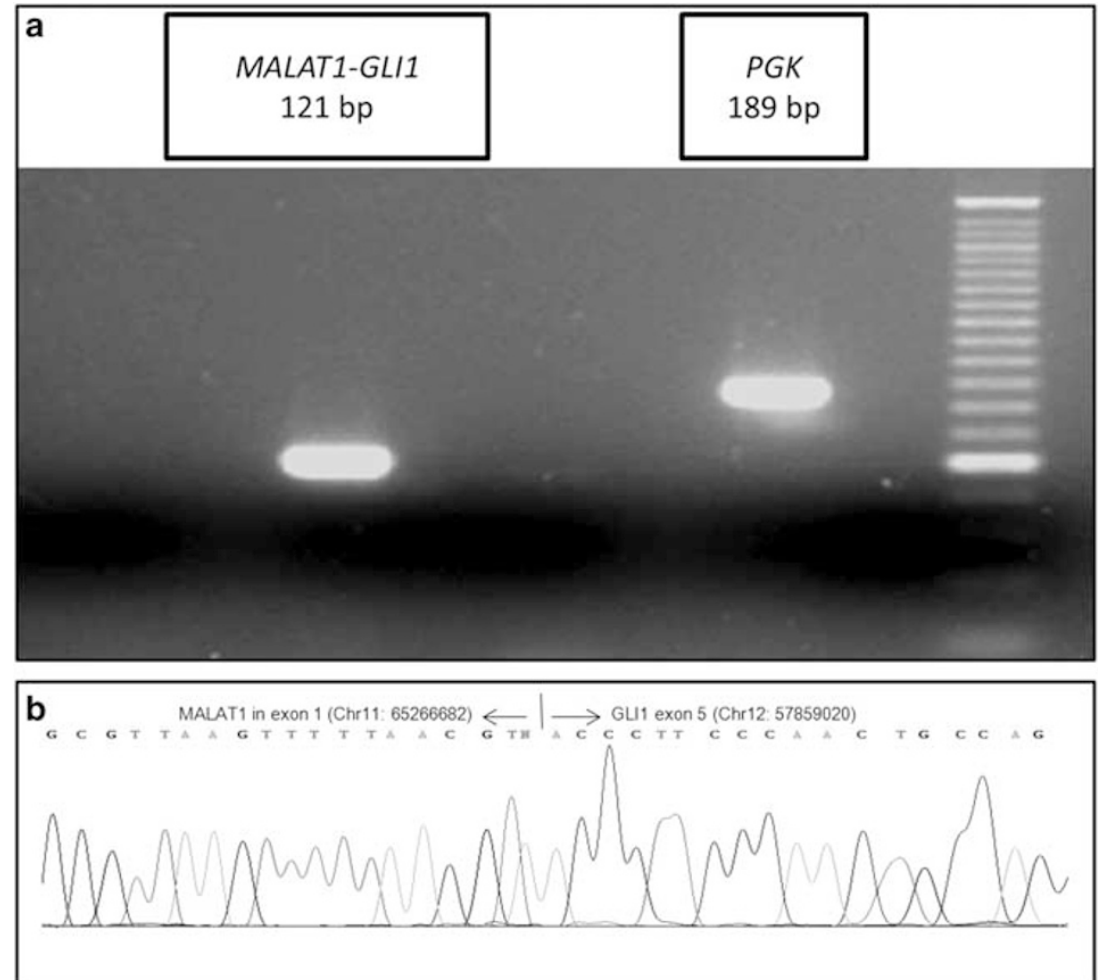

Figure 2 (a) RT-PCR-confirmed MALAT1-GLI1 fusion transcript in this case of gastroblastoma, and (b) sequencing of the cDNA-confirmed fusion of MALAT1 to GLI1.

of expression data from 369 gastric carcinomas in The Cancer Genome Atlas (TCGA) ${ }^{12}$ revealed two cases $(<1 \%)$ with overexpression of GLI1. This affirms that GLI1 IHC may be a useful screen for gastroblastoma.

\section{Gene Expression Profiling}

Gene expression profiling was successfully performed in formalin-fixed paraffin-embedded tissue from two of three gastroblastomas, three desmoidtype fibromatosis, five leiomyomas, two plexiform fibromyxomas, two biphasic synovial sarcomas, two Wilms' tumors, and three Sonic Hedgehog-type medulloblastomas. Unsupervised clustering of the expression data showed the gastroblastomas to cluster together, and to show the greatest similarity to Sonic Hedgehog-type medulloblastomas. Both of these tumor types were distinct from the other studied tumors, including the plexiform fibromyxomas (Figure 5).

\section{Discussion}

In 2009, Miettinen et al. ${ }^{1}$ described a novel biphasic gastric neoplasm of young adults, termed 'gastroblastoma'. The morphological features of these tumors varied little from case to case, and were essentially identical to those of the tumors in the present series. Although little has been previously known about the molecular genetic pathogenesis of gastroblastoma, the results of our study confirm the existence of gastroblastoma as a distinct entity, and show that they represent translocation-associated tumors, characterized by the presence of a somatic, recurrent, oncogenic MALAT1-GLI1 fusion gene. With the exception of plexiform fibromyxoma (discussed in greater detail below), this fusion gene appears to be specific to gastroblastoma, as we did not identify it in any other studied tumor, and as it has not been previously identified in any of the 369 gastric carcinomas by evaluated by RNASeq and reported in TCGA. ${ }^{12,13}$ It would thus seem that detection of MALAT1-GLI1 fusion gene by D-FISH would be very valuable ancillary test for the confident diagnosis of gastroblastoma.

Our data also suggest that immunohistochemistry for GLI1 may be useful for the diagnosis of gastroblastoma, as this protein is consistently expressed in gastroblastoma but not potential morphological mimics. The immunophenotype of gastroblastoma is otherwise nonspecific, typically consisting chiefly of keratin and CD56 expression in the epithelial component, and vimentin, smooth muscle actin, and/ or CD10 expression in the spindled cells.

MALAT1, previously called Alpha, is a long noncoding RNA and is not translated to protein. ${ }^{14}$ It is however highly expressed in the nucleus of most cells. ${ }^{14}$ Its translocation to GLI1 is expected to drive overexpression of GLI1. Our data confirm this, with GLI1 mRNA and protein overexpression in all 

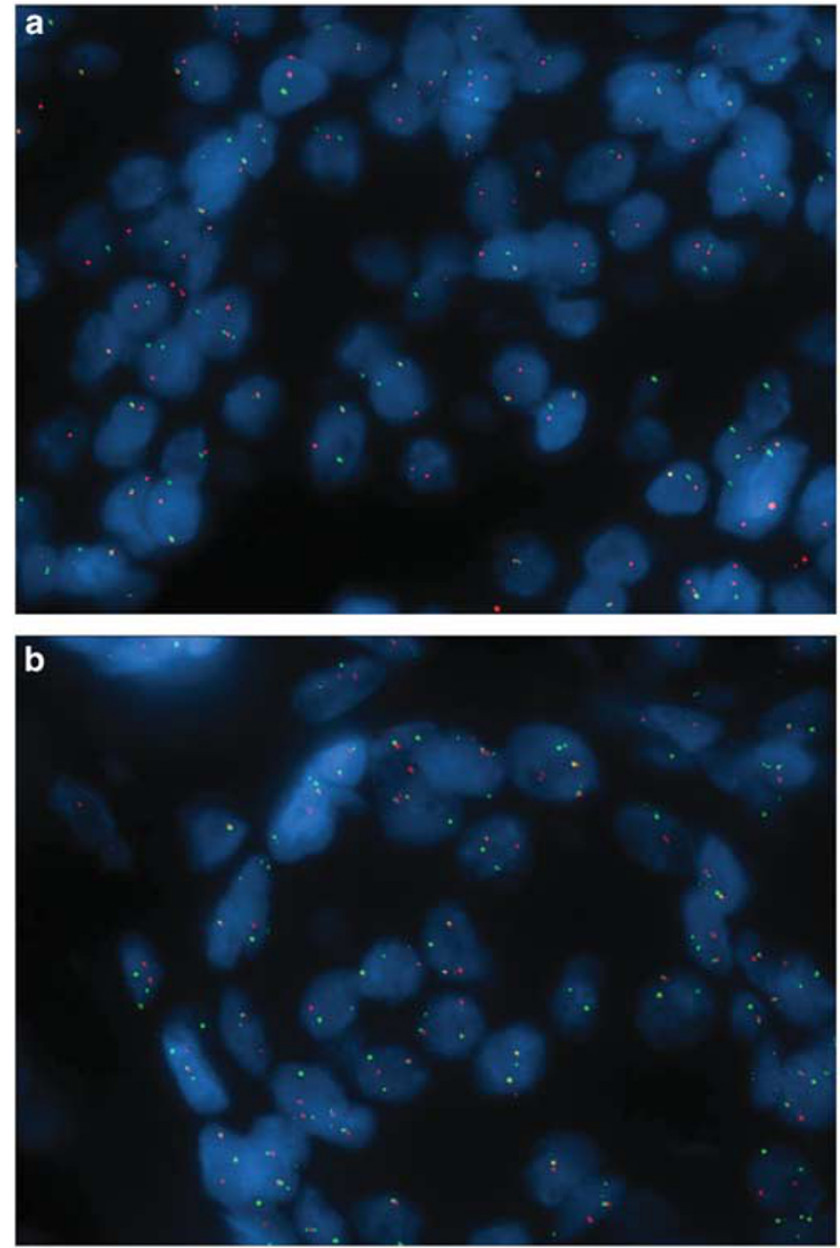

Figure 3 (a) GLI1 break-apart FISH was positive in four of four cases of gastroblastoma as shown in this panel by separation of red and green signals with one intact fusion signal (yellow) in the tumor nuclei. (b) MALAT1-GLI1 dual-fusion FISH was also positive in four of four cases of gastroblastoma as shown by fusion (overlapping) of red and green signals to give rise to yellow signals. The resulting signal pattern includes two fusion signals along with separate red and green signals.

studied cases of gastroblastoma. Our data suggest that this overexpressed GLI1 is functional (ie, transcriptionally active), as evidenced by overexpression of several of its known downstream targets with key roles in tumorigenesis-PTCH1, ${ }^{15-17}$ SOX2 ${ }^{18}{ }^{2}$ VEGFA ${ }^{19}$ and CCND $1 .{ }^{20} \mathrm{PTCH} 1$ is involved in cell proliferation and organization, ${ }^{21,22}$ SOX2 is a pro-proliferative protein involved in cellular pluripotency, ${ }^{23,24}$ VEGFA is a pro-angiogenic molecule, ${ }^{25}$ and CCND1 is involved in the cell cycle. ${ }^{26}$

GLI1 is a key protein in the Sonic Hedgehog pathway, ${ }^{27}$ and pathway analysis demonstrated upregulation of the Sonic Hedgehog pathway and downregulation of the Sonic Hedgehog antagonistic protein kinase A pathway ${ }^{27}$ in our cases of gastroblastoma. The similarity of the gene expression profile of gastroblastoma to Sonic Hedgehog-type medulloblastoma is consistent with these observations. Taken together, these data raise the intriguing
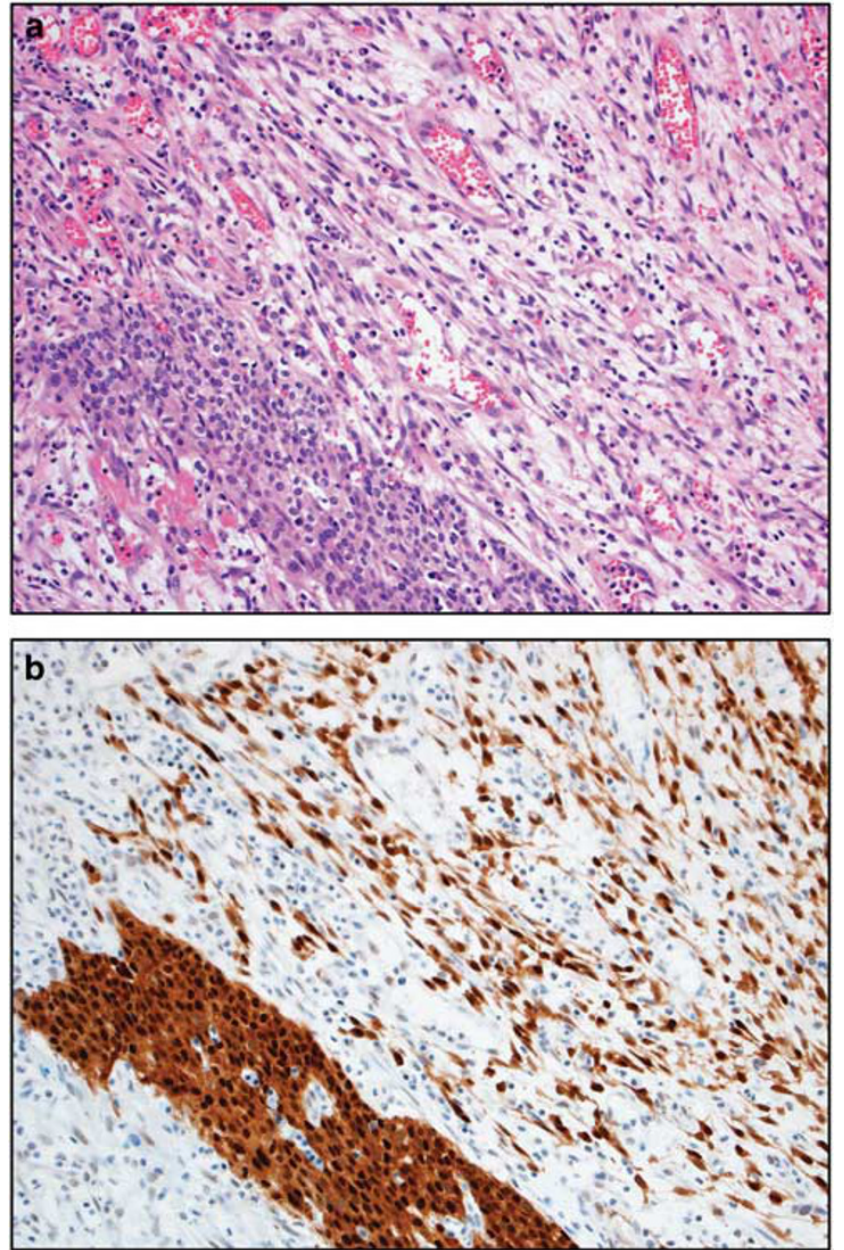

Figure 4 (a) Epithelial and spindled areas in this example of gastroblastoma are shown (case 3). (b) Both components show strong and diffuse nuclear and cytoplasmic expression of GLI1 protein.

possibility that Sonic Hedgehog pathway inhibitors may be effective targeted forms of therapy in patients with locally advanced or metastatic disease.

Both of the MALAT1-GLI1 fusion gene structures in our cases of gastroblastoma include GLI1 exons 6-12 and a novel transcription start site in exon 6. The DNA-binding zinc finger domains of GLI1 are encoded by exons 7-10 and the transactivation domain is encoded by exon 12. As such, the key domains of GLI1 are retained in the novel fusion gene (Supplementary Figure).

Plexiform fibromyxoma is another recently described, rare gastric tumor that usually occurs in young patients, and is known to harbor the MALAT1GLI1 fusion gene in a subset of cases. ${ }^{7}$ The structure of the MALAT1-GLI1 fusion gene reported in the subset of plexiform fibromyxoma is the same as that seen in gastroblastoma. ${ }^{7}$ The fusion gene is believed to have the same functional consequence in both tumor types where it is present. This, of course, raises the question of whether gastroblastoma and plexiform fibromyxoma represent related entities, or even possibly 


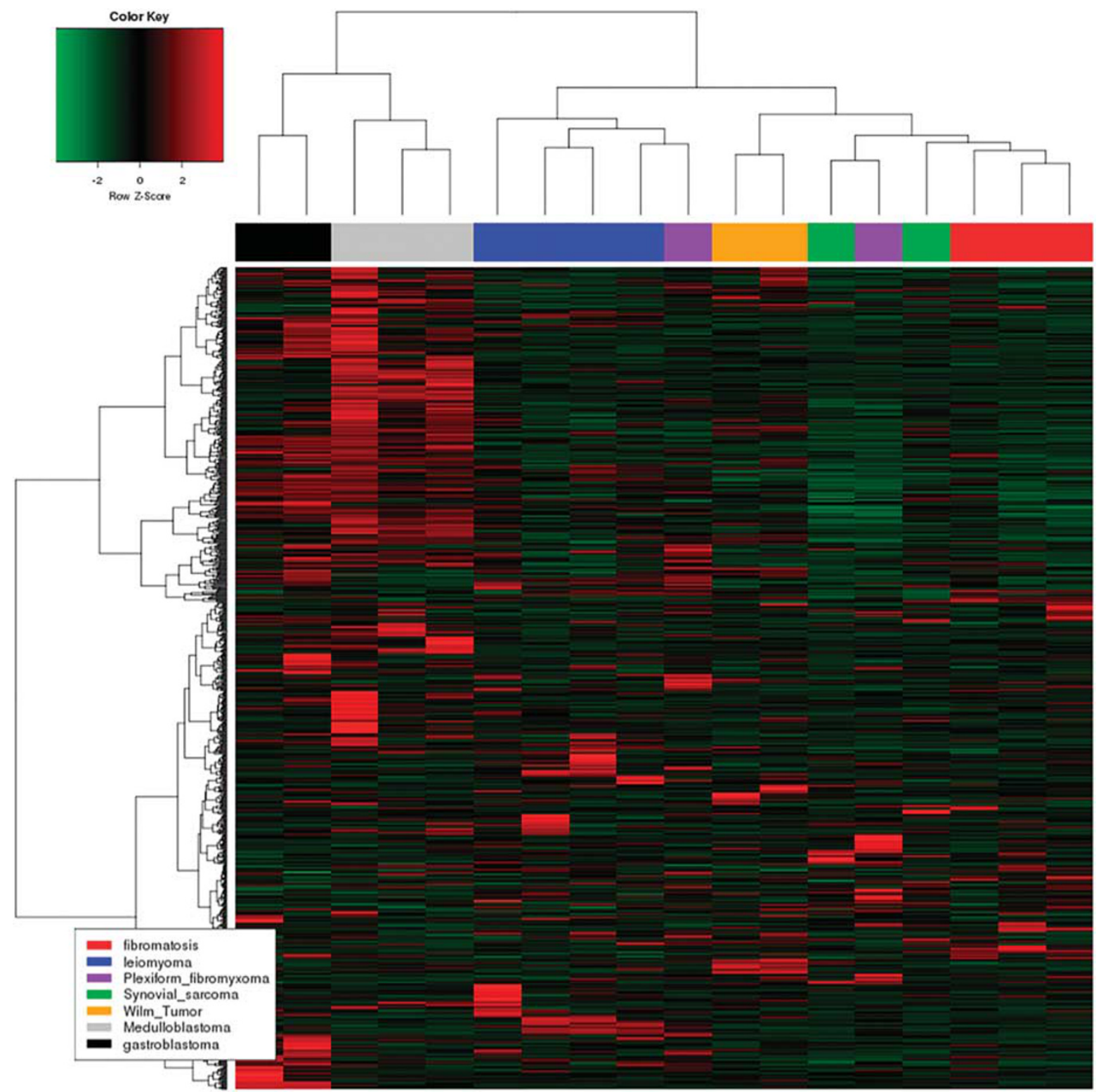

Figure 5 This heat map illustrates that by gene expression profiling gastroblastomas group together apart from other tumor types but with close similarity to Sonic hedgehog-type medulloblastoma.

different manifestations of the same entity. However, plexiform fibromyxoma is clinically benign and lacks biphasic morphology, quite different from gastroblastoma. ${ }^{7,28-36}$ We strongly suspect that the presence of MALAT1-GLI1 fusions in both of these tumors represents simply another example of identical genetic events in unrelated neoplasms, akin to the PAX3-FOXO1 or PAX3-NCOA1 fusions that may be seen in biphenotypic sinonasal sarcomas and alveolar rhabdomyosarcomas, ${ }^{37,38}$ or the EWSR1ATF1 fusions seen in clear cell sarcoma and angiomatoid fibrous histiocytoma (among others). ${ }^{39-41}$

In conclusion, we have identified a recurrent oncogenic fusion gene in gastroblastoma, MALAT1GLI1. Despite the extreme rarity of this tumor, the distinctive clinical, morphological, and molecular genetic features of gastroblastoma strongly support its classification as a unique entity. Awareness of this unusual entity, careful morphological study, and ancillary testing for the MALAT1-GLI1 fusion, GLI1 rearrangement, and/or GLI1 protein overexpression should allow for the confident diagnosis of gastroblastoma, and its distinction from various morphological mimics.

\section{Acknowledgments}

We are grateful for funding support from the Division of Anatomic Pathology, Mayo Clinic and to Janis Donovan and Shari Bartlett for excellent logistical support. We thank the pathologists who referred 
these cases in consultation and in so doing made this study possible. We thank Dr HongZheng Ren for preparing all samples used for the RNASeq analysis. This work was supported in part by funds from the Biomarker Discovery Program Center for Individualized Medicine to JJ and HZR.

\section{Disclosure/conflict of interest}

The authors declare no conflict of interest.

\section{References}

1 Miettinen M, Dow N, Lasota J, et al. A distinctive novel epitheliomesenchymal biphasic tumor of the stomach in young adults ('gastroblastoma'): a series of 3 cases. Am J Surg Pathol 2009;33:1370-1377.

2 Shin DH, Lee JH, Kang HJ, et al. Novel epitheliomesenchymal biphasic stomach tumour (gastroblastoma) in a 9-year-old: morphological, ultrastructural and immunohistochemical findings. J Clin Pathol 2010;63:270-274.

3 Wey EA, Britton AJ, Sferra JJ, et al. Gastroblastoma in a 28-year-old man with nodal metastasis: proof of the malignant potential. Arch Pathol Lab Med 2012;136: 961-964.

4 Ma Y, Zheng J, Zhu H, et al. Gastroblastoma in a 12year-old Chinese boy. Int J Clin Exp Pathol 2014;7: 3380-3384.

5 Fernandes T, Silva R, Devesa V, et al. AIRP best cases in radiologic-pathologic correlation: gastroblastoma: a rare biphasic gastric tumor. Radiographics 2014;34: 1929-1933.

6 Wei J, Xu C, Tai Y. Clinicopathologic features of gastroblastoma. Zhonghua Bing Li Xue Za Zhi 2016;45: 66-68.

7 Spans L, Fletcher CD, Antonescu CR, et al. Recurrent MALAT1-GLI1 oncogenic fusion and GLI1 up-regulation define a subset of plexiform fibromyxoma. J Pathol 2016;239:335-343.

8 Kim D, Salzberg SL. TopHat-Fusion: an algorithm for discovery of novel fusion transcripts. Genome Biol 2011;12:R72.

9 Davila JI, Fadra NM, Wang X, et al. Impact of RNA degradation on fusion detection by RNA-seq. BMC Genomics 2016;17:814.

10 Graham RP, Jin L, Knutson DL, et al. DNAJB1-PRKACA is specific for fibrolamellar carcinoma. Mod Pathol 2015;28:822-829.

11 Graham RP, Garcia JJ, Greipp PT, et al. FGFR1 and FGFR2 in fibrolamellar carcinoma. Histopathology 2016;68:686-692.

12 The Cancer Genome Atlas. Available at URL http:// cancergenome.nih.gov/. Accessed January 2, 2017.

13 Gao J, Aksoy BA, Dogrusoz U, et al. Integrative analysis of complex cancer genomics and clinical profiles using the cBioPortal. Sci Signal 2013;6:pl1.

14 Ji P, Diederichs S, Wang W, et al. MALAT-1, a novel noncoding RNA, and thymosin beta4 predict metastasis and survival in early-stage non-small cell lung cancer. Oncogene 2003;22:8031-8041.

15 Nilsson M, Unden AB, Krause D, et al. Induction of basal cell carcinomas and trichoepitheliomas in mice overexpressing GLI-1. Proc Natl Acad Sci USA 2000;97: 3438-3443.

16 Dahmane N, Lee J, Robins P, et al. Activation of the transcription factor Gli1 and the Sonic hedgehog signalling pathway in skin tumours. Nature 1997;389: 876-881.

17 Agren M, Kogerman P, Kleman MI, et al. Expression of the PTCH1 tumor suppressor gene is regulated by alternative promoters and a single functional Gli-binding site. Gene 2004;330:101-114.

18 Bora-Singhal N, Perumal D, Nguyen J, et al. Gli1mediated regulation of Sox2 facilitates self-renewal of stem-like cells and confers resistance to EGFR inhibitors in non-small cell lung cancer. Neoplasia 2015;17: 538-551.

19 Meerang M, Berard K, Felley-Bosco E, et al. Antagonizing the Hedgehog pathway with vismodegib impairs malignant pleural mesothelioma growth in vivo by affecting stroma. Mol Cancer Ther 2016;15: 1095-1105.

20 Hegde GV, Munger CM, Emanuel K, et al. Targeting of sonic hedgehog-GLI signaling: a potential strategy to improve therapy for mantle cell lymphoma. Mol Cancer Ther 2008;7:1450-1460.

21 Jia Y, Wang Y, Xie J. The Hedgehog pathway: role in cell differentiation, polarity and proliferation. Arch Toxicol 2015;89:179-191.

22 Ingham PW. The patched gene in development and cancer. Curr Opin Genet Dev 1998;8:88-94.

23 Lai YS, Chang CW, Pawlik KM, et al. SRY (sex determining region Y)-box2 (Sox2)/poly ADP-ribose polymerase 1 (Parp1) complexes regulate pluripotency. Proc Natl Acad Sci USA 2012;109:3772-3777.

24 Feng R, Wen J. Overview of the roles of Sox2 in stem cell and development. Biol Chem 2015;396: 883-891.

25 Neufeld G, Cohen T, Gengrinovitch S, et al. Vascular endothelial growth factor (VEGF) and its receptors. FASEB J 1999;13:9-22.

26 Stacey DW. Cyclin D1 serves as a cell cycle regulatory switch in actively proliferating cells. Curr Opin Cell Biol 2003;15:158-163.

27 Gorojankina T. Hedgehog signaling pathway: a novel model and molecular mechanisms of signal transduction. Cell Mol Life Sci 2016;73:1317-1332.

28 Duckworth LV, Gonzalez RS, Martelli M, et al. Plexiform fibromyxoma: report of two pediatric cases and review of the literature. Pediatr Dev Pathol 2014;17: 21-27.

29 Miettinen M, Makhlouf HR, Sobin LH, et al. Plexiform fibromyxoma: a distinctive benign gastric antral neoplasm not to be confused with a myxoid GIST. Am J Surg Pathol 2009;33:1624-1632.

30 Sing Y, Subrayan S, Mqadi B, et al. Gastric plexiform angiomyxoid myofibroblastic tumor. Pathol Int 2010; 60:621-625.

31 Kim A, Bae YK, Shin HC, et al. Plexiform angiomyxoid myofibroblastic tumor of the stomach: a case report. J Korean Med Sci 2011;26:1508-1511.

32 Lee PW, Yau DT, Lau PP, et al. Plexiform fibromyxoma (plexiform angiomyxoid myofibroblastic tumor) of stomach: an unusual presentation as a fistulating abscess. Int J Surg Pathol 2014;22:286-290.

33 Li P, Yang S, Wang C, et al. Presence of smooth muscle cell differentiation in plexiform angiomyxoid myofibroblastic tumor of the stomach: a case report. Int J Clin Exp Pathol 2014;7:823-827. 
34 Sakamoto K, Hirakawa M, Atsumi K, et al. A case of gastric plexiform fibromyxoma: radiological and pathological findings. Jpn J Radiol 2014;32:431-436.

35 Kane JR, Lewis N, Lin R, et al. Plexiform fibromyxoma with cotyledon-like serosal growth: a case report of a rare gastric tumor and review of the literature. Oncol Lett 2016;11:2189-2194.

36 Quero G, Musarra T, Carrato A, et al. Unusual focal keratin expression in plexiform angiomyxoid myofibroblastic tumor: a case report and review of the literature. Medicine (Baltimore) 2016;95:e4207.

37 Fritchie KJ, Jin L, Wang X, et al. Fusion gene profile of biphenotypic sinonasal sarcoma: an analysis of 44 cases. Histopathology 2016;69:930-936.

38 Huang SC, Ghossein RA, Bishop JA, et al. Novel PAX3NCOA1 fusions in biphenotypic sinonasal sarcoma with focal rhabdomyoblastic differentiation. Am J Surg Pathol 2016;40:51-59.

39 Kao YC, Sung YS, Zhang L, et al. EWSR1 fusions with CREB family transcription factors define a novel myxoid mesenchymal tumor with predilection for intracranial location. Am J Surg Pathol 2017;41: 482-490.

40 Panagopoulos I, Mertens F, Debiec-Rychter M, et al. Molecular genetic characterization of the EWS/ATF1 fusion gene in clear cell sarcoma of tendons and aponeuroses. Int J Cancer 2002;99:560-567.

41 Waters BL, Panagopoulos I, Allen EF. Genetic characterization of angiomatoid fibrous histiocytoma identifies fusion of the FUS and ATF-1 genes induced by a chromosomal translocation involving bands 12q13 and 16p11. Cancer Genet Cytogenet 2000;121:109-116.

Supplementary Information accompanies the paper on Modern Pathology website (http://www.nature.com/ modpathol) 